\title{
PERVASIVE TECHNOLOGIES FOR THE REDUCTION OF DISASTER CONSEQUENCES: OPPORTUNITIES AND QUESTIONS
}

\author{
VINCENZO FERRARA \\ Department of Information Engineering, Electronics and Telecommunications (DIET) \\ Sapienza University of Rome, Italy.
}

\begin{abstract}
The paper aims at put in evidence as spreading of a better and efficient technology can decrease the extreme consequences of a disaster in scenarios such as avalanches or collapsed buildings. Therefore, it is an opportunity that increases the probability of successful rescue. At the same time involves questions that may have consequences on the same mode of operation of the measuring instruments, practically by hiding or preventing their capability of detection. A review of detection methods belonging to emerging technology shows these aspects along with the questions that arise. On the other hand, rescuers need specialized instrumentations, which are available to all times, easily accessible and manageable, and that meet stringent requirements in terms of detection accuracy, quick location and reduction of false alarms. An improved technology allows reaching a satisfactory quality level of the detection method, but it must be obtained limiting spending, because a more expensive price generally decreases the availability of that instrumentation for rescuers. This aspect of availability is to be emphasized because it could create delays in the performance of the rescue practices. Recent disasters that occurred in Italian region show this as a critical point. Other important aspects are the manageability and easy operation. In order to solve efficiently the detection, actual systems integrate different techniques, often based on hybrid technologies: electronics, electromagnetics, and so on. These detection systems need an accurate management of single parts and of their mutual interaction. Complexity of technical solution must not get worse the practical use. Moreover, size and weight reduction of electronic components, with which the device is built, increase the transportability of detection system, e.g. on board of an unmanned aerial vehicle. This has consequence on an augmentation of their employment, with the opportunity to use them in scenarios characterized by high-risk rescue operations.
\end{abstract}

Keywords: disaster monitoring and mitigation, emergency preparedness, preparedness and training.

\section{INTRODUCTION}

Technological progress makes available the tools that offer more valid and effective solutions in cases of post-disaster interventions. Above all, may increase the rate of survivors allowing faster and more precise interventions, an opportunity that increases the probability of rescue. There are now available tools that allow various environmental analysis, by checking the status of real-time areas, as well as other sensors allow you to monitor the vital signs of trapped individuals. The quantity and quality of information take a specific weight, according to the shortness of the time available to carry out the rescue of people in danger of life. In fact, while the detection of vital signs is evaluated positively when it has available a relatively long time in order to intervene, the same one is a given that many would not be used when the survival time is very short, and there may be uncertainty of the data detected. In any case, it is true the rescuers need specialized instrumentations, available to all times, easily accessible and manageable, and that meet stringent requirements in terms of detection accuracy, quick localization, and reduction of false alarms.

In the second chapter, we introduce different technologies and methods for detecting buried victims during the emergencies. The chapter examines two major categories of rescue scenarios and then of detection methods that must be distinguished in the case of presence of: 
active or passive targets. That is, the detection by using active devices and detection of passive targets.

The third chapter examines some aspects of the dissemination of technology.

\section{TECHNOLOGY FOR DETECTING BURIED VICTIMS IN EMERGENCY}

The trapped victims and/or buried by rubble because of a natural or manmade disaster are among the most disturbing events and strong emotional impact. The organisation of the rescue has as priority the detection and saving of victims. First between all targets. The relief efforts must still be made in safety, to prevent accidents to rescuers, and this may cause a slowdown of operations. Unsafe building structures as a result of an earthquake, jeopardizing the lives of rescuers, prevent de facto checking of relevant areas to search for victims. At the same mode, the motion along a mountain slope run over by avalanche is particularly arduous and dangerous, due to the presence of snow mixed with bulky slabs of ice. Often, fastest results could be obtained if the rescuers have available more efficient instrumentations, suitable for the particular scenario. Although a specific instrumentation can be useful for most of emergencies, to be an effective detection in all of particular scenarios, should at least change the detection procedure.

However, there are many examples of different detection methods that use the same basic technology. Usually the scenario is the one that imposes a particular detection technique. Two major categories of rescue scenarios and then of detection methods must be distinguished: the presence of active or passive targets. They are considered active targets all those objects that produce a steady flow of information, detectable in a consistent manner from suitable sensors. The nature of the 'message' can be different: electromagnetic, sound, and so on. Belong to this category: transceivers, geophones, etc. Also fall into this category all those electronic appliances not directly designed for the detection, but that can be localized thanks to their electromagnetic emissions.

In any case, situations that are more frequent show passive targets that not transmit any useful data for the detection. Or rather, we need to organize the detection without the aid of radiation sources, worn by humans or in their proximity. Therefore, after a list of principal issues and relative solutions generally adopted for detecting in the two situations, we will discuss principally the case of passive state of the targets.

\subsection{Detection by using active devices}

The technologies based on the use of active devices are more frequent when persons are conscious of to what the environment where they live is dangerous. This is typical of many outdoor sports activities, such as off piste skiing or mountain hike in winter. But a similar methodology is adaptable for localizing objects used daily by persons, like smartphones, electronic watches, and all electronic or electromagnetic devices that should modify in a controlled mode the electromagnetic radiation that comes from a transmitter managed by a rescuer. Examining at first the case corresponding to the use of devices dedicated to perform the localization of avalanche victims, every skier or hiker brings with it a transceiver.

\subsubsection{Avalanche beacon}

Normally the device, named Avalanche Beacon or ARTVA, works as transmitter which operates at $457 \mathrm{kHz} \pm 80 \mathrm{~Hz}$ international standard frequency, ETSI standard EN 300718 . The transmitting frequency is suitable for penetrating through some meters of snow layers, sufficient to localizing persons in the most of these disasters. 
Digital beacons that show higher performance are also equipped with another transmitter at a frequency referred to as W-Link. This second transmitter is for resolving of multiple, complex burial situations by better differentiating individual transceivers. It helps the operational management of rescue by avoiding that more operators focus simultaneously on the identification of a single subject and they forget about the remaining buried victims. Moreover, the W-link allows to transmit and to receive additional data including wearer's vital signs or identification. The transmitting frequency is different for region. European countries adopt a frequency fixed to $869.8 \mathrm{MHz}$, United States and Canada establish frequencies in the range $916 \div 926 \mathrm{MHz}$.

The wave at the frequency of $475 \mathrm{kHz}$ is about but not perfectly omnidirectional and consists of a signal arranged as a train of low-power pulses. The pulse width is in the interval $70-900 \mathrm{~ms}$, with a duty-cycle in the range 10\%-69\%. The ARTVAs worn by hikers buried under snow, after they are run over by avalanche, continue to transmit. The remaining other hikers, which were not affected by the same avalanche, switch the ARTVA device in the receiver mode, and then they start to locate each buried person. Instrument determines the direction of a transmitter evaluating the maximum value of power receiving signal. That is, identifying the exact path by means of the antenna radiation pattern, designed to be selective in angle, i.e. suitably directional. The detectable signal is a function of the used ARTVA, analogue or digital, in that the two models have a different limit on maximum distance allowable between the two transmitting and receiving antennas, for a proper detection. Generally, for a digital device the maximum distance is about $10 \mathrm{~m}$. Moreover, digital devices use three antennas in order to manage the detection of multi targets, evaluating for each both direction and distance of the buried transmitters. Wireless data of the W-link channel, which come from other ARTVAs switched in search mode, contribute to determine location of buried person faster and with greater precision.

When all skiers bring Avalanche Beacon, each of them could assume the function of rescuer, with the advantage to start immediately the research. In effect, the survival time of the victims under avalanche decreases quickly from $90 \%$ to $30 \%$ if rescuers remove a person after one hour. In order to complete the search on entire area, where the avalanche has invested people, it suggests a specific procedure, which takes into account the useful link budget NLOS (no line of sight) of the sensors. That is, the distance within which we have certainty of the detection, even in the worst orientation of buried transmitter. Such a search procedure is differentiated by number of researchers at work and for advancement phases of the localization, coarse and precise. Figure 1 shows the suggestion for the optimum path during the coarse phase in the case of only one researcher (a) and of many (b). The path must trace lines characterized by mutual distance equal to double of the useful link budget NLOS (d). A minor distance increases total time of the monitoring. Using a distance greater, the risk of not detecting some people is large.

An alternative to ARTVA transceivers usage is to wear passive reflectors, named Recco® reflectors, which operate as tuned resonant circuits, when an electromagnetic radiation affects them. Normally a skier wears two reflectors, by including them into each jacket's sleeve or inside the two boots. The main advantage is the absence of battery. The more evident issue is the uncertainty that the few skiers fully equipped of necessary transceiver can be between the survivors. Otherwise, there will be a big delay for the start of the rescue. Although this alternative uses passive reflectors, the basis functioning principle is the same of previous one, which adopts ARTVA, but with lower efficiency. The frequency of transmitted signal is equal to $917 \mathrm{MHz}$. Passive reflector retransmits the wave doubling its frequency, and consequently the receiver of the transceiver operates at a frequency of $1834 \mathrm{MHz}$. 


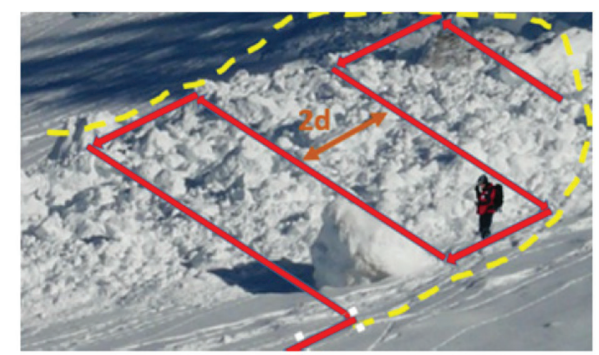

(a)

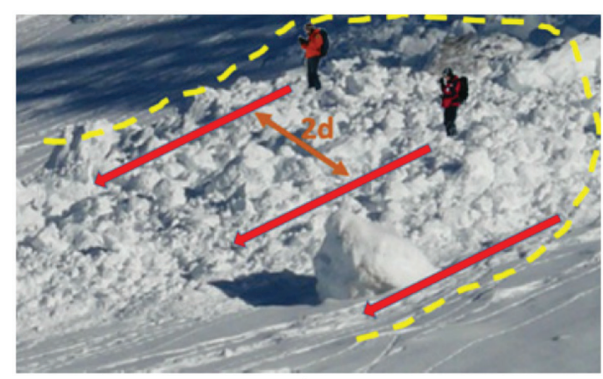

(b)

Figure 1: Suggestion for the optimum path, during the coarse phase, in the case of only one researcher (a) and of many (b).
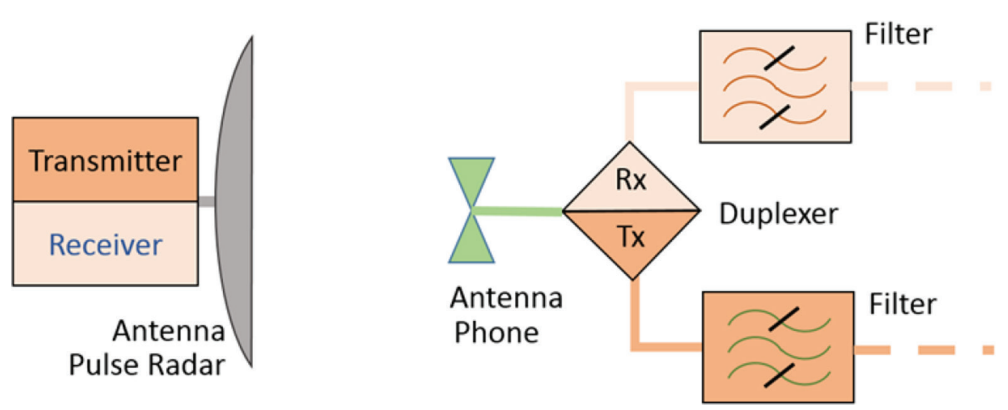

Figure 2: General setup for the detection of RF-devices even if defective or broken off.

\subsubsection{Radio frequency device detection}

In all different situations from the activities who plan to face high-risk environments, people do not wear special devices to ensure an efficient localization, in anticipation of a possible disaster. The search for persons that bring personal electronic devices, like smartphone, radio controlled watches, and so on, i.e. all electronic device that operate at radio frequency (RF), can be assisted with pulse radar systems [1]. As the Recco® reflector, such devices can be exposed to a radar pulse electromagnetic wave. If this pulse is near to resonance frequency of filters included in the RF-devices, the generated echo could be used for locating the owner person. In the particular case of defective smartphone, and with reference to the Fig. 2, a radar pulse is sent to buried and broken device, the antenna of phone receives impulse, and 
signal is forwarded through the duplexer, a high-Q filter used to separate the transmit and receive frequencies, to the filter that selects among the frequencies contained in the signal.

If the spectrum of pulse is in the range of RF filter bandwidth, the energy is stored in the filter. When it switches off the transmitting signal, i.e. the radar pulse is no longer transmitted, the stored energy generates a RF echo that propagates over duplexer and to transmitting antenna of smartphone. Finally, the radar can detect this RF echo in the same tuned band.

Unfortunately, it is not certain that the victims bring these electronic devices or objects are close to the body. However, it is a valid alternative when other methods fail for adverse environmental conditions. Better, this technology should be integrated and to cooperate together with different detecting systems in order to resolve many hard situations.

\subsection{Detection of passive targets}

As emphasized previously, when as a result of a disaster you must locate buried victims, the most common situation is that of individuals without electronic devices worn, neither those designed for the purpose of their detection, nor the generic RF devices, usable for the occasion like pseudo-coherent re-transmitters. Their position will only happen if one or some physical, biological or biomechanical characteristics of buried people are clearly identifiable with respect to similar physical and mechanical characteristics of the environment that surrounds them. Technology must accentuate the differences and/or have a high sensitivity to be able to identify individuals and avoid false alarms.

\subsubsection{Geophones}

A possibility is to detect the sounds emitted for example by the persons calling help. There are examples in the literature of sound detection systems manufactured using a network of geophones [2] or a single tri-axial geophone [3], in order to detect vital signs as heartbeat, breathing, and footstep. Actually, most of these systems are dedicated to discover persons hidden in closed environments, e.g. clandestine immigrants concealed in truck trailers. In effect, the main use is in the field of security, in the surveillance tasks. The main issue of this detection technique is the requirement of a very quiet environment, hard to find during rescue and emergency activities.

\subsubsection{Thermography}

The thermal inspections could be another alternative. It uses infrared (IR) radiations, in the range of wavelength around from one to ten micron. At the basis of this method, the well-known law of Stefan-Boltzmann, concerning black body emittance. Equation 1 is its extension to real body, where $\mathrm{q}, \varepsilon, \sigma$, and $\mathrm{T}$ are respectively: the emittance of a black body, emissivity of real body, Stefan Boltzmann constant, equal to $5.67 \cdot 10^{-8} \mathrm{~W} \mathrm{~m}^{-2} \mathrm{~K}^{-4}$, and the absolute temperature.

$$
q=\varepsilon \cdot \sigma \cdot T^{4}
$$

This method, also used in civil engineering, for the state of building structures surveys, provides results that are strongly influenced by environmental conditions, such as sunlight, rain and wind. For an acceptable quality of the detection images it is also necessary that there is a minimum of thermal difference between target and objects of the surrounding environment that are at direct contact, a minimum temperature difference of the order of $10^{\circ} \mathrm{C}$. These conditions are best found in the cases of people buried under rubble. Unfortunately, the depth of penetration through the debris layers is very limited and this constitutes a major impediment to the detection. Variants that make use of the stress of the material layers by means of 
microwaves to raise the contrast of the image photogrammetric [4], through analysis of the evolution of temperature profiles during the heating and cooling phases, are normally used to locate cold objects, and not the human beings.

Moreover, in the mountain environment should be observed that you add to the adverse environmental conditions, the physiological mechanism of the rapid reduction of the temperature of the human body in the process of frostbite under the snow and ice. Note, since the radiation manifests a poor depth of penetration, the result of detection normally has origin from heat of the subcutaneous layers, and minimally by the deeper organs.

\subsubsection{Ground penetrating radar}

We can summarize the operation of a GPR system as follows:

- GPR system transmits an electromagnetic wave into the depths of the soil or other structure that is obstacle to the detection of objects buried or hidden;

- the different value of permittivity $\left(\varepsilon_{\mathrm{r}}\right)$ between adjacent layers changes the wavelength of the radiation and its propagation velocity in the medium;

- also the relative magnetic permeability $\left(\mu_{\mathrm{r}}\right)$ and the electrical conductivity $(\sigma)$ have influence on the characteristics of the electromagnetic wave parameters;

- the change in the electromagnetic properties between two contiguous layers generates a new scattered wave that can reach the receiving antenna of the GPR system;

- the received wave allows to calculate the distance of the surface that produced the scattered wave;

- other assessments, such as the relative movements between GPR and target, can be obtained by using specific types of GPR systems.

However, we should emphasize that the structures mainly made of metallic materials or reinforced concrete slabs are electromagnetic transmission media with losses, which reduce the depth of penetration achievable and create deleterious multiple reflections.

GPR systems operate with frequencies in the range from a few tens of $\mathrm{MHz}$ to some $\mathrm{GHz}$, i.e. they show a larger wavelength than the IR radiation, and penetrate more deeply into the materials. Therefore, they are more suitable for the purpose of the research of buried individuals. The depth reached depends on the type of GPR system and by the material that constitutes the transmission medium. In general, a frequency increase improves resolution but reduces the penetration depth. The GPR systems are primarily distinguished by the domain design choice: in time or frequency, see Fig. 3. In time domain, the operating

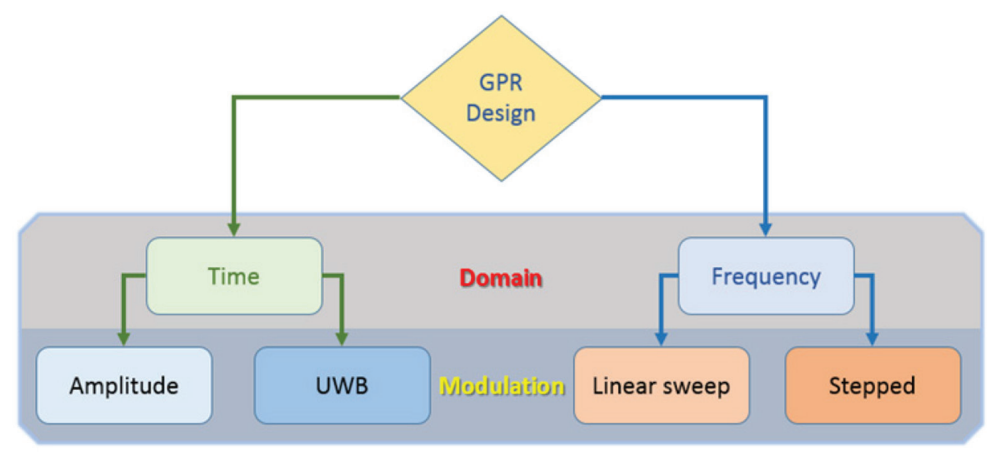

Figure 3: Different GPR designs. 
principle is to send a pulse into the ground and then listen to the backscattered echoes. Main categories of time domain systems are two: the amplitude modulated and the carrier free or UWB GPR. Actually, the term UWB (Ultra-Wide Band) slightly differs from that of the conventional air radar systems, defining UWB GPR those systems with signals that have a fractional bandwidth $\left(\right.$ Fract $\left._{B W}\right)$ of eqn. 2, where BW is the bandwidth and $f_{\text {centr }}$ the central frequency, larger than $100 \%$.

$$
\text { Fract }_{B W}=\frac{B W}{f_{\text {centr }}} \cdot 100 \% \geq 100 \% .
$$

The first type, i.e. the amplitude modulation, sends a pulse with the carrier frequency, which in turn is modulated by a square wave. The pulse width determines the vertical resolution: more the pulse is narrow, the better the resolution. Note the UWB GPR has been developed to get systems with widths of much larger bands.

In the frequency domain, you modulate a continuous wave $(\mathrm{CW})$ by main two modes: linear sweep and stepped. A linear sweep generates frequency-modulated continuous-wave (FMCW), and you can carry out different shapes of the modulation such as at saw tooth and triangular. Obviously, if you change the frequency of the $\mathrm{CW}$ in fixed steps, the modulation is named stepped frequency continuous wave (SFCW).

The FMCW setup of Fig. 4 demonstrates how the distance between target and GPR is evaluated. A control voltage with ramp shape modifies the frequency of the oscillator (VCO). A splitter divides amplified waveform by sending two identical signals, one toward transmitting antenna and other to mixer. The scattered wave from the target, with a fractional amount of energy, arrives to the receiving GPR antenna. A low noise amplifier (LNA) increases the signal magnitude at proper value for the mixing operation. The mixer generates output signals of frequency equal to the sum and to the difference of the frequencies of input. The filters select the signals with frequency difference, which are then amplified. These signals are significant of the distance that exists between target and GPR system, like eqn. 3 expresses. In the equation, $\mathrm{D}$ is the searched distance, $v$ is velocity of electromagnetic wave in the transmission medium, $\Delta \mathrm{f}$ is frequency difference between transmitted and received signals, and $d f l d t$ is frequency shift per unit time.

$$
D=\frac{v \cdot \Delta f}{2 \cdot\left(\frac{d f}{d t}\right)} .
$$

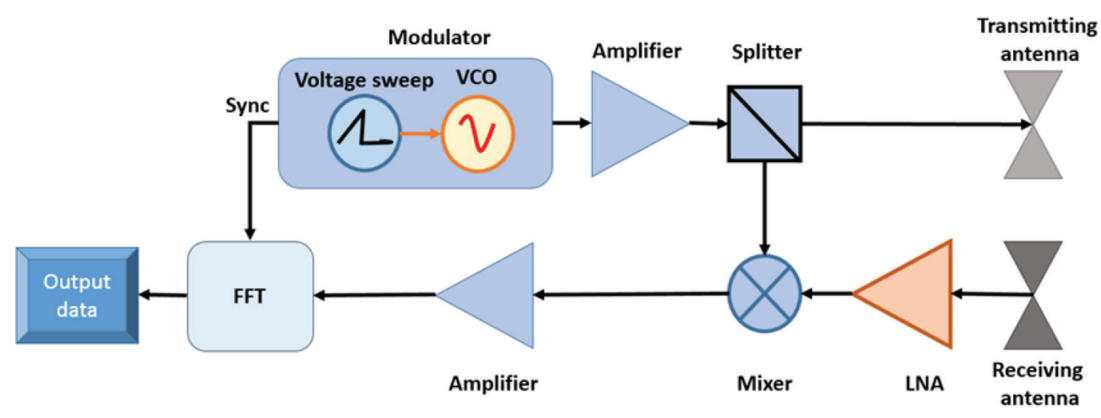

Figure 4: Block diagram of FMCW GPR. 
In the case of triangular shape, the measure of Doppler frequency $f_{D}$ can be implemented by evaluating two frequencies difference $\Delta \mathrm{f}_{1}$ and $\Delta \mathrm{f}_{2}$ at the rising and falling edges respectively, as you can see from eqn. 4. Doppler frequency is useful for detecting movement of a target, e.g. vital signs of a buried person.

$$
f_{D}=\frac{\left|\Delta f_{1}-\Delta f_{2}\right|}{2} .
$$

Examples of cheap design of FMCW GPR are in literature [5].

From the point of view of the false alarms, the main source is the non-stationary clutter. However, there are other different noise sources, such as stationary clutter, internal noise, random jitter, and narrowband jamming. Some researchers proposed interesting solutions that solve problems arising from non-stationary clutter. In particular, Zaikov and Sachs designed a prototype UWB radar that allows the removal of that clutter together with signal to clutter separation, by principal component analysis [6]. The problem of false alarms is an important issue, which need to be resolved especially for the case emergency scenario. This is because, in such a scenario are more frequent the noises as motion of objects located near the GPR, and rescue engines, together with radio interferences added, like smartphones [7].

\section{A WIDESPREAD TECHNOLOGY SUCH AS EFFECTIVE SOLUTION}

All the technologies examined in the previous chapter have been selected for their noninvasive feature. An essential quality for not exposing the rescue workers to the dangers, which generally follow a disaster. However, among the listed technologies, those that can be used for detecting and locating people buried without personal devices, dedicated to the detection and/or exchange of information with the search system, are of major interest for personnel rescue. In fact, rescuers work directly in the field, in various situations and environmental conditions and prefer to use stand-alone instruments, showing a direct result, not conditioned by the presence/absence of devices owned of the victims. Among these technologies, those using the GPR systems result from what has been said the most appropriate in the case of people buried or trapped. Both for the nature of the electromagnetic radiation, which allows a greater depth of penetration compared to that based for example on thermography, and for the possibility to operate in noisy environments, commons in emergency scenarios, choosing the most appropriate type of GPR, and realizing a suitable cancellation technique or at least a reduction of noisy effects. Therefore, in the following of the paper we will refer on GPR systems, to distinguish detection techniques and the methodologies you can use.

\subsection{Dissemination of the technology}

At first, it is appropriate to make some observations on the relationship between technological advancement, in terms of goals achieved and perspectives, and actual use of technologically advanced instruments. It often occurs that, even if the innovative instrument has passed the post prototypal phase, and could be available for use, it is rarely used. The origin of this problem is manifold, but essentially: cultural and economic.

The cultural problem can be expressed by a dissemination of scientific knowledge and innovation limited to the circle of the only researchers and experts. Sometimes the application versatility of new devices or algorithmic methods is not appreciated until after a long process. The awareness of this problem has reached the top institutions, particularly European ones, which have launched in recent years many international programs of collaboration, e.g. the 
COST Actions. The main purpose of them is the exchange of technological information at various levels, university, industrial and state agencies. At corollary of these collaborations have been undertaken a series of initiatives aimed at the dissemination of core technologies and expertise to young people.

The economic aspect often represents a ballast to the spread of complete systems, or simple devices, technologically advanced in the institutions responsible for security and civil protection. Fortunately, in recent times it has been observed that the technological progress feeds himself. That is, the progress made in nanotechnology has led to a considerable reduction in costs. Thus the realization of a FMCW radar system at the ISM band performed using integrated RF components has a cost of implementation which is also less than a tenth of an equivalent realized employing components housed in rugged unibody chassis.

The considerable reduction in the size of the devices and consequently of the entire system has other important consequences: the reduction of the energy required for its operation, which is accompanied by the decrease of the voltage values involved, and the considerable weight reduction. The implications are different. The characteristic low voltage-low power increases the energy autonomy of the system that can best be designed for use in mobility. To reach even to the complete autonomy, using harvesting techniques in order to recover all the energy needed for its operation from the surrounding environment. The reduction of the weight means greater manageability of the system, but it is also a prerequisite to the design of higher performance systems that can solve difficult operational situations.

It has already been said that the characteristic of the non-invasive research systems is important for safety purposes. However, if the monitoring of an area run over by an avalanche is made by dragging of the detection system over the snow and the slabs of ice, the danger to the rescuers persists. Similarly, in the case of collapsed buildings, where to move among the rubble poised it is extremely dangerous. In such cases, the adoption of self-propelled robot carrying the sensor or the mounting on board UAVs of the same monitoring systems is possible and opportune. In the case of on board of UAV GPR, the monitoring technique is more complex [8] and requires the integration of the aircraft's trim measures. These ones first of all serve to correctly interpret the pointing direction of the GPR antenna and to calculate the position of the target detected by applying the necessary fixes to the aircraft coordinates. This arrangement provides greater monitoring speeds with all the benefits that come with it.

A policy of greater diffusion of technology, and technological culture, could allow the achievement of regional centres of more efficient monitoring, with powers to intervene faster and more targeted. The search for a solution, or a technological tool for control of the area at risk, it would not be the result of initiatives forced by the necessities of the last hour, close to the event disastrous. In fact, it should be taken into account even the appearance of bureaucratic slowness, which slow down exceedingly purchases and the installation of important systems and especially needed. In recent events in Italy, for example, only the good will and abnegation of researchers volunteers allowed the rescuers to operate in relative safety, by implanting a radar control to check on the looming danger of avalanches.

\section{REFERENCES}

[1] Loschonsky, M., Feige, Ch., Rogall, O., Fisun, S. \& Reindl, L.M., Detection technology for trapped and buried people, IEEE MTT-S International Microwave Workshop on Wireless Sensing, Local Positioning, and RFID, pp. 1-6, 2009.

https://doi.org/10.1109/imws2.2009.5307879 
[2] Ascione, M., Buonanno, A., D’Urso, M., Vinetti, P., Angrisani, L. \& Schiano Lo Moriello, R., A method based on passive acoustic sensors for detection of vital signs in closed structures. Instrumentation and Measurement Technology Conference (I2MTC), IEEE International, pp. 1764-1769, 2012. https://doi.org/10.1109/i2mtc.2012.6229551

[3] Vinod Reddy, V., Divya, V., Khong, Andy W.H. \& Ng, B.P., Footstep detection and denoising using a single triaxial geophone. Circuits and Systems (APCCAS), 2010 IEEE Asia Pacific Conference on Circuits and Systems, pp. 1171-1174, 2010. https://doi.org/10.1109/apccas.2010.5775004

[4] Di Marzio, C.A., Rappaport, C.M., Wen, L. \& Sauermann, G.O., Microwave-enhanced infrared thermography. Proceedings of SPIE, 3710, pp. 173-179, 1999.

[5] Ferrara, V., Troiani, F., Frezza, F., Mangini, F., Pajewski, L., Simeoni, P. \& Tedeschi, N., Design and realization of a cheap ground penetrating radar prototype @ $2.45 \mathrm{GHz}$. IEEE Browse Conference Publications - 2016 10th European Conference on Antennas and Propagation (EuCAP), pp. 1-4, 2016. https://doi.org/10.1109/eucap.2016.7482008

[6] Zaikov, E. \& Sachs, J., UWB radar for detection and localization of trapped people, eds Boris Lembrikov, Ultra Wideband, InTech, 2010. https://doi.org/10.5772/10004

[7] Sachs, J., Helbig, M., Herrmann, R., Kmec, M., Schilling, K., Zaikov, E. \& Rauschenbach, P., Trapped victim detection by pseudo-noise radar. Proceedings of ACWR '11, 1st International Conference on Wireless Technologies for Humanitarian Relief, pp. 265-272, 2011. https://doi.org/10.1145/2185216.2185289

[8] Fruehauf, F., Heilig, A., Schneebeli, M., Fellin, W. \& Scherzer, O., Experiments and algorithms to detect snow avalanche victims using airborne ground-penetrating radar. IEEE Transactions On Geoscience and Remote Sensing, 47(7), pp. 2240-2251, 2009. https://doi.org/10.1109/tgrs.2009.2012717 